\title{
Sensory Nerve Conduction Study with Inching Test in Palmar Digital Neuropathy
}

\author{
Tatsuya Ueno ${ }^{1}$, Masayuki Baba ${ }^{1}$, Akira Arai $^{1}$, Chieko Suzuki ${ }^{2}$ and Masahiko Tomiyama ${ }^{2}$
}

\begin{abstract}
:
Mild palmar digital neuropathy may be underestimated because selective nerve conduction studies (NCS) of the palmar digital nerve are challenging. We herein report two cases of palmar digital neuropathy. We performed sensory NCS in each finger using the standard approach. Both cases showed a decrease in the amplitude of sensory nerve action potential (SNAP) in the localized finger. Furthermore, the sensory nerve inching test identified the lesion site. When performing NCS in patients with finger sensory impairment, we recommend recording the SNAP in each finger using standard NCS at the wrist, as well as sensory nerve inching testing.
\end{abstract}

Key words: palmar digital nerve, neuropathy, nerve conduction study, sensory nerve inching test, trauma

(Intern Med 60: 469-472, 2021)

(DOI: 10.2169/internalmedicine.5514-20)

\section{Introduction}

The palmar digital nerves (PDNs) originate from the sensory terminal branch of the median and ulnar nerves (1). Although several causes, such as tendon sheath abnormalities, joint disorders, vasculitic neuropathies, and nerve tumors, may damage the PDNs, trauma is the most common etiology (1). Kumar et al. reported that traumatic neurapraxia in digital nerve injuries of the hand has a favorable prognosis (2). However, palmar digital neuropathy may be underestimated because of the lack of data in the literature on the incidence of digital nerve neurapraxia (2).

As the clinical diagnosis of a digital neuropathy is often evident, a nerve conduction study (NCS) is seldom required (1). However, NCS is useful for determining the entrapment site, assessing the severity, predicting the prognosis of the disease, and conducting patient education. Furthermore, whether or not their symptoms will improve is an important patient concern. Selective stimulation of PDNs has been challenging because of technical issues, such as innervation overlaps and volume conduction artifacts $(3,4)$. A standard NCS may not be effective in identifying palmar digital neuropathy (4). Therefore, additional methods are re- quired for the confirmation in clinical practice. These NCS techniques are essential for patient education and the provision of informed consent in patients with finger sensory impairment.

We herein report two cases of palmar digital neuropathy confirmed by sensory nerve conduction studies (SCS) performed and recorded for each finger using standard NCS with stimulation at the wrist. In addition, the sensory nerve inching test was useful for identifying the lesions in both cases.

\section{Materials and Methods}

The NCS was performed with an electromyography machine (Dantec Keypoint software program, version 5.13; Alpine BioMed, San Carlos, USA) using a surface recording electrode and a bipolar surface electrode for stimulation. The sensitivity was set at 5 to $20 \mu \mathrm{V} / \mathrm{div}$ with a $20-$ to $3,000-\mathrm{Hz}$ bandpass. The patient's skin temperature was measured using a thermometer (Thermofocus-Pro; Tecnimed Srl, Vedano Olona, Italy) and confirmed to be above $32^{\circ} \mathrm{C}$. Sensory nerve action potentials (SNAPs) were recorded by placing the surface recording electrode in the center, not the side, of each finger at the proximal and distal interphalangeal joints, set $3 \mathrm{~cm}$ apart from each other. The ground electrode was

${ }^{1}$ Department of Neurology, Aomori Prefectural Central Hospital, Japan and ${ }^{2}$ Department of Neurology, Hirosaki University Graduate School of Medicine, Japan

Received: June 3, 2020; Accepted: July 5, 2020; Advance Publication by J-STAGE: August 29, 2020

Correspondence to Dr. Tatsuya Ueno, tatsuya_ueno@med.pref.aomori.jp 
placed between the recording and stimulating sites. A bipolar surface electrode was used for stimulation. We performed antidromic stimulation of the ulnar and median nerves at the wrist. The stimulus duration was $0.2 \mathrm{~ms}$, and the stimulus intensity was gradually increased until the maximum SNAP amplitudes were achieved. All patients were clinically and electrophysiologically assessed to rule out carpal tunnel syndrome (CTS) (Table).

Table. Nerve Conduction Studies (Lesion Side).

\begin{tabular}{lcc}
\hline \multicolumn{1}{c}{ Motor nerve } & Case 1 & Case 2 \\
\hline LMN- DL (ms) & 3.2 & 4.2 \\
LMN- CMAP (distal/proximal) (mV) & $5.7 / 5.0$ & $5.1 / 4.6$ \\
LMN- MCV (m/s) & 58.8 & 56.5 \\
LMN (2nd lumbricales)- DL (ms) & 3.4 & 3.8 \\
LUN (1st palmar interosseous)- DL (ms) & 3.3 & 3.4 \\
Sensory Nerve & & \\
LMN (ring finger)- DL (ms) & 2.7 & 2.9 \\
LUN (ring finger)- DL (ms) & 2.5 & 2.6 \\
\hline
\end{tabular}

L: left, MN: median nerve, UN: ulnar nerve, DL: distal latency, CMAP: compound muscle action potential, MCV: motor nerve conduction velocity

Normal values:

MN DL $<4.2 ;$ MN CMAP $>3.5 ;$ MN MCV $>48$.

$\mathrm{UN}$ DL $<3.4$; UN CMAP > 2.7; UN MCV >49.

\section{Case Reports}

\section{Case 1}

A 74-year-old Japanese woman with a history of left breast cancer resection 3 years earlier complained of a tingling sensation in the left thumb and index finger. She reported shoveling snow continuously for two months. She was suspected of having CTS and received steroid injections into the carpal tunnel. However, her condition did not improve, and she was referred to our department. Neurological examinations revealed mild hypesthesia at the ulnar side of the left thumb and the radial side of the left index finger. Tinel's sign was positive in the thenar $4 \mathrm{~cm}$ distal from the distal carpal crease. She had no atrophy or weakness of the left hand.

We performed antidromic stimulation of the ulnar and median nerves at the wrist and recorded the SNAPs in each finger. A median SCS at the wrist revealed a decreased SNAP amplitude in the left thumb and index finger (Fig. 1A). An antidromic sensory nerve inching test in the index finger showed conduction delay at the lateral palmar divisions of the median nerve (PDMN) (Fig. 1B). She was diagnosed with neuropathy at the lateral PDMN owing to palm compression caused by shoveling snow. She was ad-

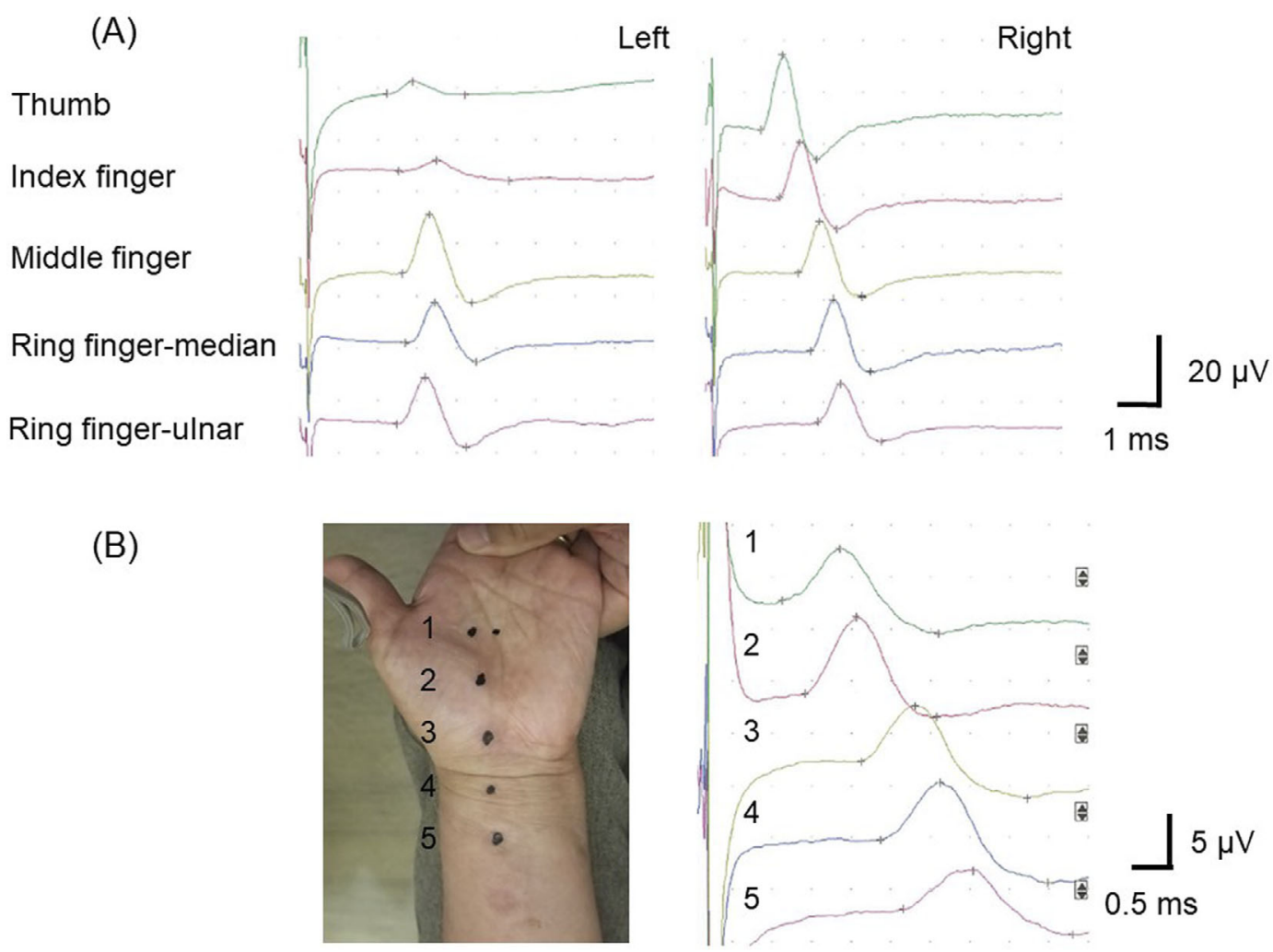

Figure 1. (A) A median sensory nerve conduction study at the wrist showed decreased amplitudes of sensory nerve action potentials in the left thumb $(4.8 \mu \mathrm{V})$ and index finger $(5.6 \mu \mathrm{V})$ compared with those in the right thumb $(33 \mu \mathrm{V})$ and index finger $(26 \mu \mathrm{V})$. (B) Antidromic sensory nerve inching test, stimulating 5 points at intervals of $2.5 \mathrm{~cm}$. The inching test demonstrated a conduction delay between the "2" and " 3 " stimulations. 
(A)

Thumb

Index finger

Middle finger

Ring finger-median

Ring finger-ulnar
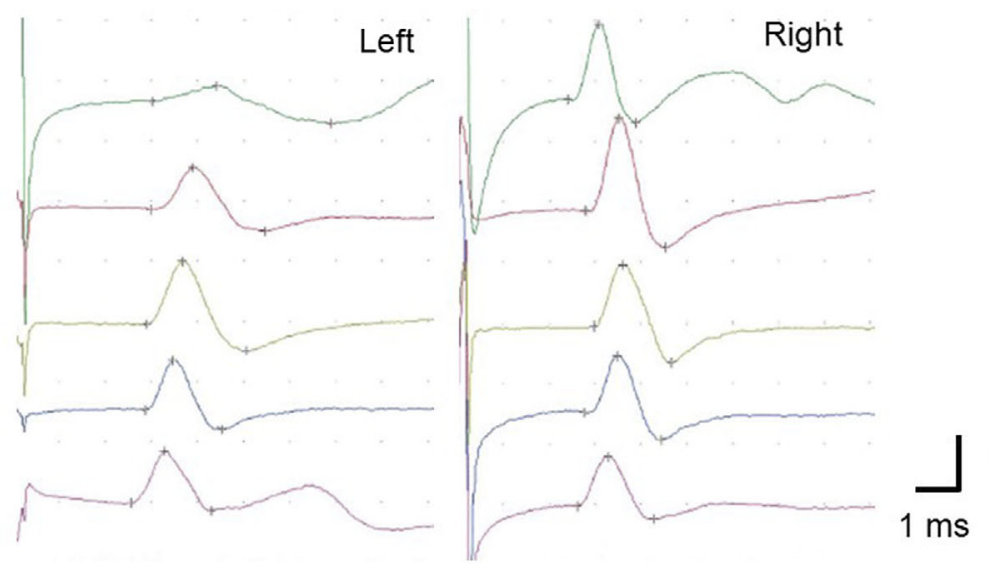

$20 \mu \mathrm{V}$

(B)

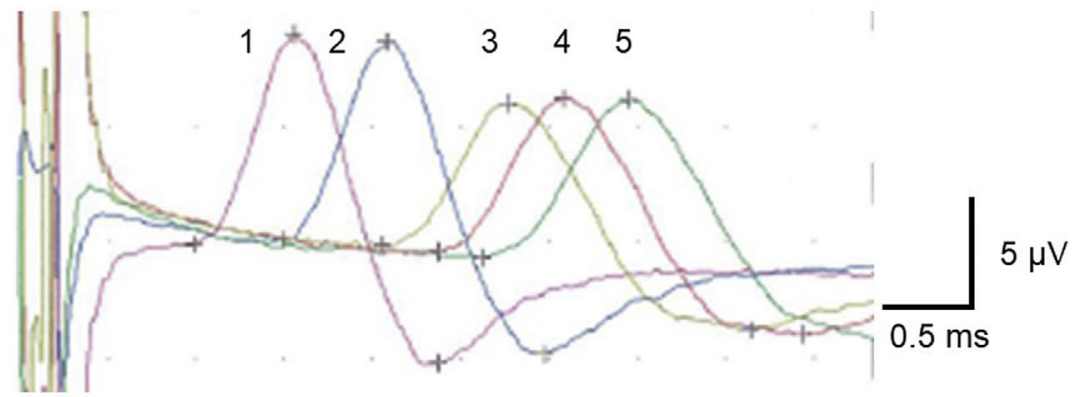

Figure 2. (A) A median sensory nerve conduction study at the wrist showed decreased amplitudes of sensory nerve action potentials in the left thumb $(7.8 \mu \mathrm{V})$ and left index finger $(17 \mu \mathrm{V})$ compared with those in the right thumb $(28 \mu \mathrm{V})$ and index finger $(38 \mu \mathrm{V})$. (B) Antidromic sensory nerve inching test, stimulating 4 points $(1,2,3$, and 5$)$ at intervals of $2.5 \mathrm{~cm}$. Stimulation " 4 " was performed at the midpoint between " 3 " and " 5 " to confirm whether the waveform was correct. The inching test demonstrated decreased amplitudes of sensory nerve action potentials with conduction delay at the lateral palmar divisions of the median nerve between $5 \mathrm{~cm}$ and $7.5 \mathrm{~cm}$ distal to the distal carpal crease (stimulation 3).

vised to avoid excessive palm compression, and her symptoms improved after three months.

\section{Case 2}

A 22-year-old man presented with a 2-week history of numbness and tingling in the left thumb and left index finger. He was a police officer who had begun riding a police motorcycle only two weeks before the onset of his symptoms. Cervical magnetic resonance imaging (MRI) was unremarkable. He was referred to our department for an electrophysiological evaluation under suspicion of CTS. Neurological examinations revealed hypesthesia on the ulnar side of the left thumb and the radial side of the left index finger. He had no thenar atrophy, ring finger splitting, Phalen's sign, or Tinel's sign at the wrist. However, Tinel's sign at the thenar was positive. An NCS was performed as in Case 1. A median SCS at the wrist revealed decreased SNAP amplitude in the left thumb and index finger (Fig. 2A). Furthermore, an antidromic sensory nerve inching test performed in the index finger showed a decreased SNAP amplitude with conduction delay at the lateral PDMN (Fig. 2B). Tinel's sign was positive at the sites where abnormalities were observed on the sensory nerve inching test. MRI of the left palm showed no abnormality. He was diagnosed with neuropathy at the lateral PDMN caused by applying pressure on the handlebars of the motorcycle during training. He was advised to wear gloves, and his symptoms improved after three months.

\section{Discussion}

We described two cases of palmar digital neuropathy diagnosed by an SCS of each finger using standard NCS, with stimulation at the wrist. The sensory nerve inching test identified the lesion site. Based on this result, we were able to advise the patients in how to prevent further deterioration. These results suggest that this NCS technique for patients with finger sensory impairment is useful for patient education.

Although several factors, such as tendon sheath abnormalities, joint disorders, vasculitic neuropathies, and nerve tumors, can result in palmar digital neuropathy, the most common cause is trauma (1). In addition, lacerations, blunt trauma, iatrogenic damage, fracture and dislocation of digits, and external compression can also cause traumatic palmar digital neuropathies (1). Damage to common PDN and pal- 
mar proper digital nerves (PaPDNs) results in sensory loss restricted to the adjacent sides of two fingers and the side of the finger, respectively (1). Sympathetic dysfunction, such as the absence of sweating, dryness of skin, skin creases, and loss of turgor are other reliable signs of nerve injury (2).

The lesion site can be identified from a sensory assessment and careful examination for callus, swelling, points of tenderness, or Tinel's sign (1). In our cases, external compression resulted in palmar digital neuropathy. Both cases showed hypesthesia at the ulnar side of the left thumb and the radial side of the left index finger, indicating the area of the lateral PDMN; and Tinel's sign at the thenar. In both cases, Tinel's sign was positive at the abnormal site on sensory nerve inching tests. These results suggest that it may be possible to distinguish PDN from CTS by focusing on the area where Tinel's sign appears.

Seddon described three terms for describing peripheral nerve injuries: neurapraxia, axonotmesis, and neurotmesis (5). Surgical repair of digital neurapraxia lesions caused by traumatic digital nerve injuries leads to complete recovery at six to eight weeks (2). Sensory recovery among patients who do not require surgical repair for isolated digital nerve injury can take from 12 days to 6 months (6). Although NCS is seldom required, as the clinical diagnosis and cause of digital neuropathy are often obvious (1), we believe that identifying the distribution and impact of nerve injury on NCS is useful for educating and informing patients. Both of our cases had decreased SNAPs at the thumb and index finger, conduction delay, and changes in SNAPs at the PDMN without complete loss of SNAPs in the PDN, indicating axonotmesis with a certain degree of neurapraxia. We were able to predict the functional prognosis, and both patients had completely recovered by three months after the diagnosis after following our advice to avoid excessive palm compression. Therefore, recording SNAPs in each finger by standard NCS at the wrist is useful if patients have finger sensory disturbances after hand trauma. In patients with CTS, the sensory inching test with palmar stimulation has proven useful for assessing the entrapment site (7). We identified the conduction delay in the palm using the sensory nerve inching test. Thus, the sensory inching test may also be useful in cases of palmar digit nerve injury as well as CTS.

Several limitations associated with the present study warrant mention. First, although Zanette et al. reported the usefulness of more selective stimulation of PaPDNs at the webspace $(4,8)$, we performed antidromic SCS with wrist stimulation. The SNAP was evaluated with a surface recording electrode placed at the center, and not at the side, of the proximal and distal interphalangeal joints. In daily clinical practice, the recording electrode is not placed on the side of the finger. We adopted the conventional placement method in consideration of the possibility of observing unreliable waveform changes due to changes in the recording electrode position. Therefore, we were able to record SNAPs of two PaPDNs, except for the ring finger. Because this standard technique cannot separately evaluate the radial and ulnar sides of the PaPDN, we were unable to determine the injuries to a single PaPDN. This may have led to false-negative findings. Second, we did not utilize imaging techniques, such as ultrasonography, to verify the location of the lesion. The evaluation of CTS using ultrasonography has been established (9). Ultrasonography is minimally invasive and is useful for identifying and visualizing lesion sites. However, even though the lesion site could not be visualized, the possibility of a space-occupying lesion was unlikely in the present case, given the clinical course. In the future, prospective studies will be needed to evaluate PDN using ultrasonography and NCSs to identify the lesion site, severity, and prognosis.

In summary, when performing NCS for patients with finger sensory impairment, we recommend recording the SNAP in each finger using a standard NCS at the wrist and comparing the changes in the amplitude and waveform with those on the other hand. The sensory nerve inching test is useful for identifying the lesion site.

The authors state that they have no Conflict of Interest (COI).

\section{References}

1. Stewart JD. Focal peripheral neuropathies. 4th ed. JBJ publishing, West Vancouver, 2010: 348-355.

2. Kumar S, Hassouna H, Penematsa S. Clinical and user-friendly classification of traumatic digital nerve injuries of hand. Arch Orthop Trauma Surg 127: 527-530, 2007.

3. King JC, Dumitru D, Wertsch JJ. Digit distribution of proper digital nerve action potential. Muscle Nerve 24: 1489-1495, 2001.

4. Zanette G, Lauriola MF, Tamburin S. An electrodiagnostic technique for assessing palmar proper digital nerves of the hand: normative data and clinical application. Muscle Nerve 52: 972-980, 2015.

5. Seddon H. A classification of nerve injuries. Br Med J 2: 237-239, 1942.

6. Siddiqui A, Benjamin CI, Schubert W. Incidence of neurapraxia in digital nerve injuries. J Reconstr Microsurg 16: 95-98, 2000.

7. Kimura J. The carpal tunnel syndrome: localization of conduction abnormalities within the distal segment of the median nerve. Brain 102: 619-635, 1979.

8. Zanette G, Lauriola MF, Tamburin S. Electrodiagnosis of lesions of median and ulnar nerve hand sensory branches: a case series. $\mathrm{J}$ Clin Neurophysiol 33: 454-457, 2016.

9. Cartwright MS, Hobson-Webb LD, Boon AJ, et al. Evidencebased guideline: neuromuscular ultrasound for the diagnosis of carpal tunnel syndrome. Muscle Nerve 46: 287-293, 2012.

The Internal Medicine is an Open Access journal distributed under the Creative Commons Attribution-NonCommercial-NoDerivatives 4.0 International License. To view the details of this license, please visit (https://creativecommons.org/licenses/ by-nc-nd/4.0/).

(C) 2021 The Japanese Society of Internal Medicine Intern Med 60: 469-472, 2021 